\title{
Transcriptomic analysis between Normal and high-intake feeding geese provides insight into adipose deposition and susceptibility to fatty liver in migratory birds
}

Guosong Wang ${ }^{1,2+}$, Long Jin ${ }^{1 \dagger}$, Yan $\mathrm{Li}^{1}$, Qianzi Tang ${ }^{1}$, Silu Hu${ }^{1}$, Hengyong Xu' ${ }^{1}$ Clare A. Gill' ${ }^{2}$, Mingzhou Li ${ }^{1 *}$ and Jiwen Wang ${ }^{* *}$ (D)

\begin{abstract}
Background: Dysregulation of adipogenesis causes metabolic diseases, like obesity and fatty liver. Migratory birds such as geese have a high tolerance of massive energy intake and exhibit little pathological development. Domesticated goose breeds, derivatives of the wild greyleg goose (Anser anser) or swan goose (Anser cygnoides), have high tolerance of energy intake resembling their ancestor species. Thus, goose is potentially a model species to study mechanisms associated with adipogenesis.

Results: Phenotypically, goose liver exhibited higher fat accumulation than adipose tissues during fattening (liver increased by 3.35 fold than 1.65 fold in adipose), showing a priority of fat accumulation in liver. We found the number of differentially expressed genes in liver (13.97\%) was nearly twice the number of that in adipose (6.60\%). These differentially expressed genes in liver function in several important lipid metabolism pathways, immune response, regulation of cancer, while in adipose, terms closely related to protein binding, gluconeogenesis were enriched. Typically, genes like MDH2 and SCD, which have key roles in glycolysis and fatty acids metabolism, had higher fold change in liver than in adipose tissues. Three hundred two differentially expressed long noncoding RNAs involved in regulation of metabolism in liver were also identified. For example, IncRNA XLOC_292762, which was $5.7 \mathrm{~kb}$ downstream of FERMT2, a gene involved phosphatidylinositol-3,4,5-trisphosphate binding, was significantly down-regulated after the high-intake feeding period. Further investigation of documented obesityrelated orthologous genes in goose suggested that understanding the evolutionary split from mammals in adipogenesis will make goose fatty liver a better resource for future research.
\end{abstract}

Conclusions: Our research reveals that goose uses liver as the major tissue to regulate a distinct lipid synthesis and degradation flux and the dynamic expression network analyses showed numerous layers of positive responses to both massive energy intake and possible pathological development. Our results offer insights into goose adipogenesis and provide a new perspective for research in human metabolic dysregulation.

Keywords: Adipogenesis, Goose, Transcriptome

* Correspondence: mingzhou.li@sicau.edu.cn; wangjiwen@sicau.edu.cn

${ }^{\dagger}$ Guosong Wang and Long Jin contributed equally to this work

${ }^{1}$ Farm Animal Genetic Resources Exploration and Innovation Key Laboratory

of Sichuan Province, Sichuan Agricultural University, Chengdu, Sichuan

611130, People's Republic of China

Full list of author information is available at the end of the article

(c) The Author(s). 2019 Open Access This article is distributed under the terms of the Creative Commons Attribution 4.0 International License (http://creativecommons.org/licenses/by/4.0/), which permits unrestricted use, distribution, and reproduction in any medium, provided you give appropriate credit to the original author(s) and the source, provide a link to the Creative Commons license, and indicate if changes were made. The Creative Commons Public Domain Dedication waiver (http://creativecommons.org/publicdomain/zero/1.0/) applies to the data made available in this article, unless otherwise stated. 


\section{Background}

The balance of energy storage and energy expenditure is critical for normal adipose deposition and lipid metabolism. Adipose tissue has been recognized as a major endocrine organ and acts as the host for adipogenesis in mammals [1]. Excessive energy intake results in an increase in the volume and weight of adipocytes and causes dysregulation of lipid metabolism in the body [2]. Such dysregulation is reflected by variable lipid deposition in different adipose tissues, and it is usually associated with abnormal liver lipid accumulation, which can lead to steatosis and obesity [3]. In contrast to mammals, migratory birds show distinct lipid deposition patterns and use liver instead of adipose tissue as the main organ for lipid metabolism [4-6].

As a typical species domesticated from a migratory bird, goose (Anser anser or Anser cygnoides) has a completely different mechanism of lipid deposition from mammals and from some terrestrial poultry, such as chicken $[7,8]$. A goose has the capability of depositing excess lipid in its liver. Research has demonstrated that the formation of goose fatty liver shares similar phenotypic changes with human non-alcoholic fatty liver, but differs in pathological development because goose fatty liver only shows a low level of inflammation and other immune responses $[7,9,10]$. This distinct difference in the phenotype indicates that goose fatty liver might become a resource for better understanding lipid deposition in birds and for human fatty liver research. Previous research on the synthesis and delivery of fatty acids focused on goose liver without considering adipose tissues $[7,11,12]$. To explore the dynamic genetic pattern behind the regulation of lipid deposition in goose, we have built a weight-gain model to investigate the mechanism. We collected and performed RNA-seq on liver, subcutaneous adipose, and abdominal adipose to better understand the expression network. Through comprehensive analysis, we showed that goose regulates lipid metabolism differently to mammals and that liver plays the most important role in this metabolic process. We envision that goose is a model for understanding lipid metabolism.

\section{Results}

Phenotypic changes of liver and adipose tissues after high-intake feeding

Body weight increased $32.3 \%$ by the end of the fattening process $\left(p\right.$-value $=8.30^{*} 10^{-4}$, fold-change $\left.=1.32\right)$ and the high intake group was significantly heavier beginning at day 10 of the fattening period (Fig. 1a, Additional file 7). Tissues related to lipid metabolism were heavier after fattening, with liver increasing the most in relative weight by 3.35 fold ( $p$-value $=0.0011$, Fig. 1b, Additional file 8), comparing to abdominal adipose increased by 1.65 fold. Moreover, after we assessed the lipid content of liver, we found it increased drastically from $6.22 \pm 0.83 \%$ to $73.56 \pm 1.14 \%$ (Fig. 1c), which largely contributed to the increased weight of the fatty liver. Red oil staining followed by integrated optical density (IOD) also confirmed substantial lipid deposition in liver (Fig. 1c, Additional file 1).

\section{Transcriptomic difference of liver and adipose tissues after high-intake feeding}

To investigate dynamic expression changes induced by high-intake feeding, we generated an average of $\sim 11.50 \mathrm{~Gb}$ high-quality RNA-seq data. We identified an average of $77.44 \%$ protein-coding genes with FPKM $\geq 0.1$ and $1702 \mathrm{pu}$ tative lncRNAs (Most lncRNAs were sense intergenic lncRNAs (44.6\%), followed by divergent lncRNAs, and other 3 categories, Additional file 2). These IncRNAs showed similar expression characteristics with other research [13].

There were substantial differences between tissues in both mRNA profiles and lncRNA profiles (weighted average proportion variance $=0.47$ and 0.55 , respectively), followed by either interaction between treatment and tissue (weighted average proportion variance $=0.16$ in mRNA profiles) or treatment (weighted average proportion variance $=0.16$ in lncRNA profiles), indicating the major driver of the differences in expression is tissue, and the treatment effect on IncRNA expression patterns is bigger than mRNA expression patterns (Additional file 3).

Unsupervised clustering also recapitulated the distinct expression pattern between liver and adipose tissues (Fig. 2a). Tissue-dominated clustering patterns and the distinct between-group liver clustering patterns revealed the overwhelming differences between tissues, and liver was more affected by treatment. Within-group correlation between tissues also confirmed the lower correlation between adipose and liver than between two adipose tissues (Fig. 2b). Control-group sample C2-SA showed lower correlation to adipose tissues in general, we hypothesize that this sample is partially contaminated with tissues such as skin.

Protein coding genes involved in dynamic lipid metabolism We found more than 6000 DEGs between liver and adipose tissues (under both normal and high-intake feeding conditions), compared to only $\sim 400$ DEGs between the two adipose tissues. We identified 1930 DEGs in liver (13.97\% of 13,815 expressing genes with FPKM >0.1), compared to $1045(6.60 \%$ of 15,829 genes with FPKM $>0.1)$ and 891 DEGs $(4.73 \%$ of 18,839 genes with FPKM $>0.1)$ in abdominal adipose and subcutaneous adipose after high-intake feeding, respectively (Fig. 2c). The detection of more than twice the number of DEGs in liver compared to adipose, and the large number of liver-specific DEGs supports the 


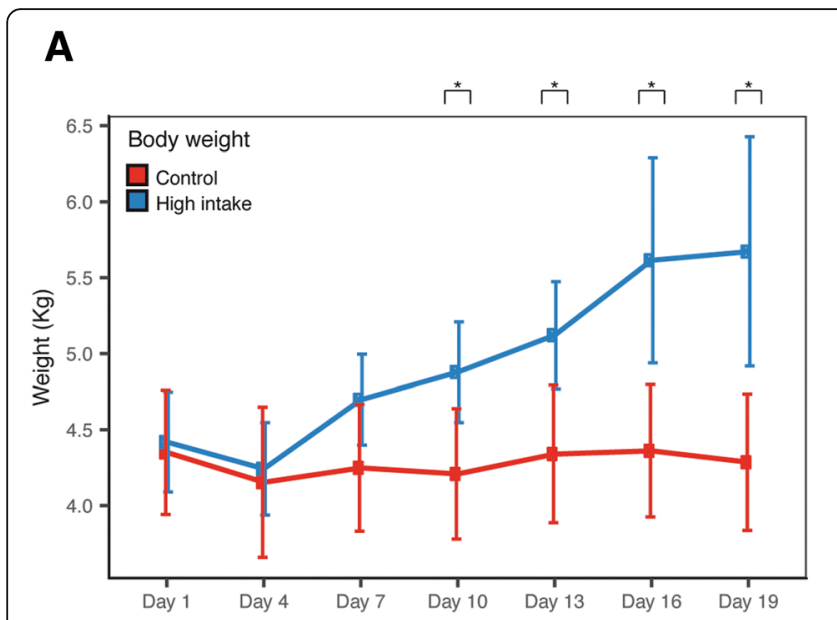

B

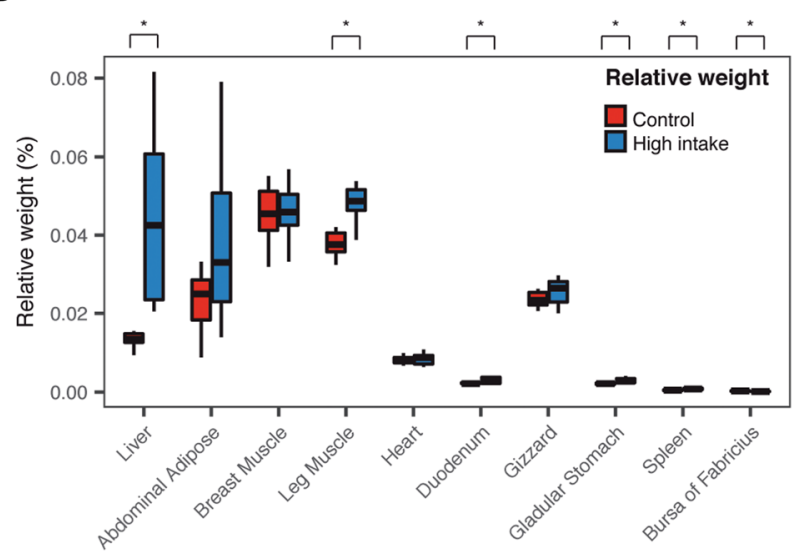

C
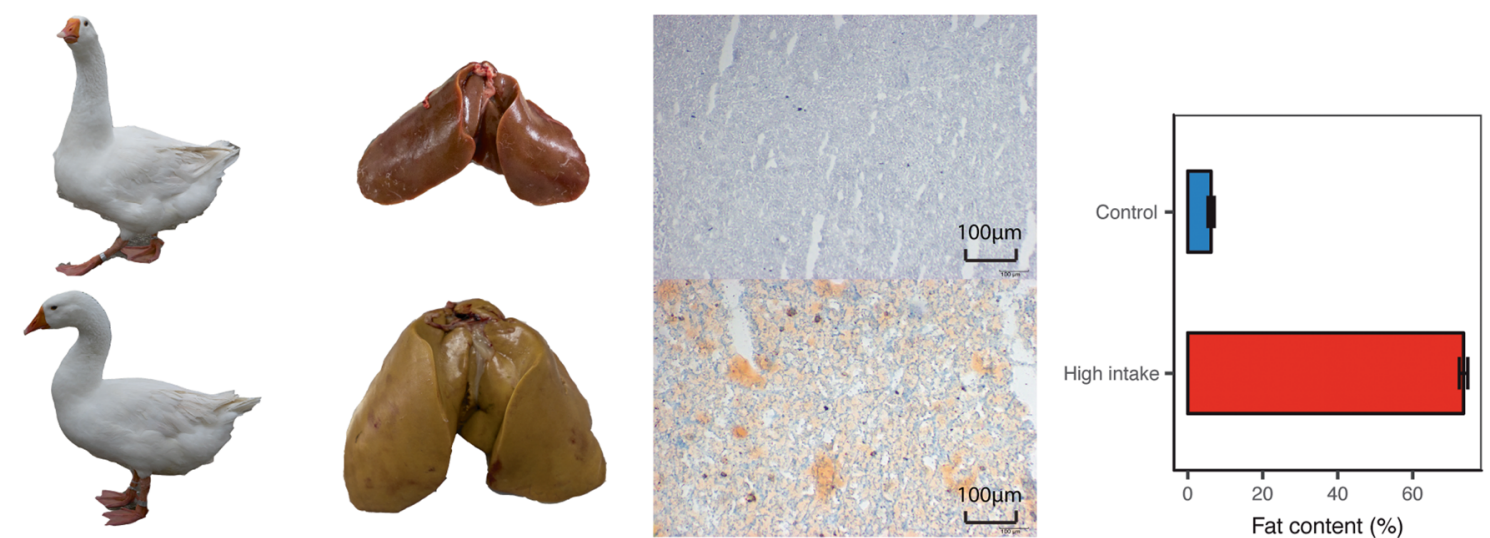

Fig. 1 Phenotypic differences between normal and high-intake fed geese. a Sequential changes in weight. b Absolute weight and relative weight of tissues. Relative weight = absolute weight/body weight. c Enlarged photos of liver, corresponding frozen sectioned red oil staining and Soxhlet extraction of lipid content of livers. Photos of geese and livers were taken from the geese used in this study

hypothesis that liver has a role in lipid metabolism during high-intake feeding.

As expected from the changes observed in phenotype, DEGs found in liver were significantly involved in metabolic pathways such as amino acids metabolism, carbon metabolism, and immune response. These genes tended to be functional in ATP binding, protein binding, oxidation-reduction process, and gluconeogenesis (Fig. 3a). Similar enrichment of DEGs found in abdominal adipose and subcutaneous adipose was observed and most of the enriched pathways and GO terms were related to metabolism as expected. The changes in metabolic processes were related to up-regulation of expression in the liver upon excess energy intake (Additional file 4). We also noticed that down-regulated DEGs were more involved in immune response, especially cancer-related pathways (Additional file 4). The association between expression changes and little or no pathological development in goose should be further investigated.

We found expression of 14 out of 20 previously identified goose mitochondria and important nuclear mitochondria-related genes [14]. The mitochondrial genes did not show a substantial response to high-intake feeding and most did not exhibit significant changes in expression in the 3 tissues we examined (Additional file 5). Gene enrichment analysis showed that nuclear genes related to the mitochondrial functions oxidation-reduction (adjusted $p$-value $=3.96^{*} 10^{-4}$ ) and mitochondrial matrix (adjusted $p$-value $=8.54^{*} 10^{-10}$ ) were significantly up-regulated (Additional file 6). Genes such as malate dehydrogenase $2(\mathrm{MDH} 2)$ was up-regulated after high-intake feeding with liver showed the biggest change (fold-change $=2.01,0.92$ and 0.94 in liver, abdominal adipose and subcutaneous adipose, respectively). The increased intensity of mitochondrial metabolism is suggestive of elevated energy production and consumption, expected from the phenotypic changes and treatment.

Long noncoding RNAs are related to fatty liver formation Differences between liver and adipose tissues were also reflected by lncRNAs. Similar to protein coding genes, more differentially expressed (DE) lncRNAs were identified in liver (302 DE lncRNAs, 19.24\% of 1570 lncRNAs with FPKM > 0.1) than adipose tissues (52 for abdominal 


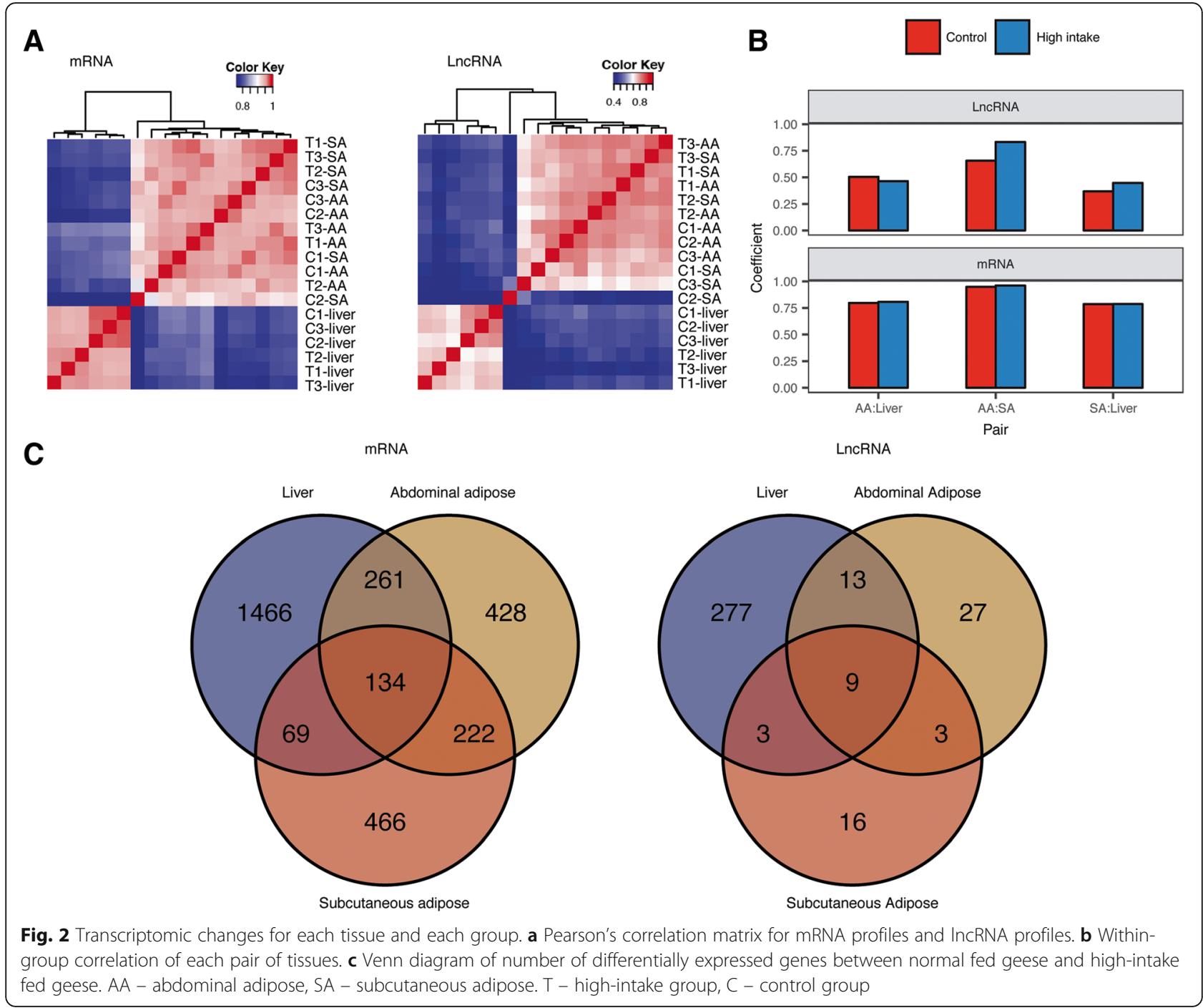

adipose, $3.13 \%$ of 1662 lncRNAs with FPKM $>0.1$ and 41 for subcutaneous adipose, $2.43 \%$ of 1689 lncRNAs with FPKM >0.1), and the majority of these DE lncRNAs were specific to liver (Fig. 2c). To determine the role of lncRNAs in the formation of fatty liver, DEGs that were highly correlated to specific lncRNAs with the highest fold change after the high-intake feeding period showed a strong relationship to metabolism. Enriched terms included amino acids metabolism, protein binding, and endoplasmic reticulum, which was found as a usual response to high-intake feeding, suggesting lncRNAs may act as possible trans-acting regulators of protein coding genes (Fig. 3b). By narrowing down to different categories of lncRNAs, strong enrichment for metabolism and immune functions was also observed within each type (Fig. 4a). Among protein coding genes spatially associated with each type of IncRNA, an average of $8.5 \%$ of them were differentially expressed. Genes involved in fatty acid metabolism, cGMP metabolic processes, and oxidation-reduction were significantly enriched. Among protein coding genes located within $10 \mathrm{~kb}$ of the IncRNA, we found the genes ZFAT, GJD2, HOXA10, and B3GALT2 (Additional file 9) that are related to metabolism, degradation of endoplasmic reticulum protein and transcription factors [15-17]. These nearby lncRNA-mRNA pairs are suggestive of cis-acting regulation of lipid metabolism during high-intake feeding. For example, FERMT2, which is involved phosphatidylinositol-3,4,5-trisphosphate binding, was possibly down-regulated by a downstream ($5.7 \mathrm{~Kb}$ ) intergenic antisense (convergent) lncRNA XLOC_292762 that was up-regulated after the high-intake feeding period (Fig. 4b). Antisense lncRNAs have been identified functional in mammalian gene network. For example, they can regulate the expression of mRNA by forming an RNA-RNA duplex at the $5^{\prime}$ end of the mRNA which is important to 5' end-dependent degradation pathways. The further exploration of antisense lncRNAs and other types of 
A

Benjamini
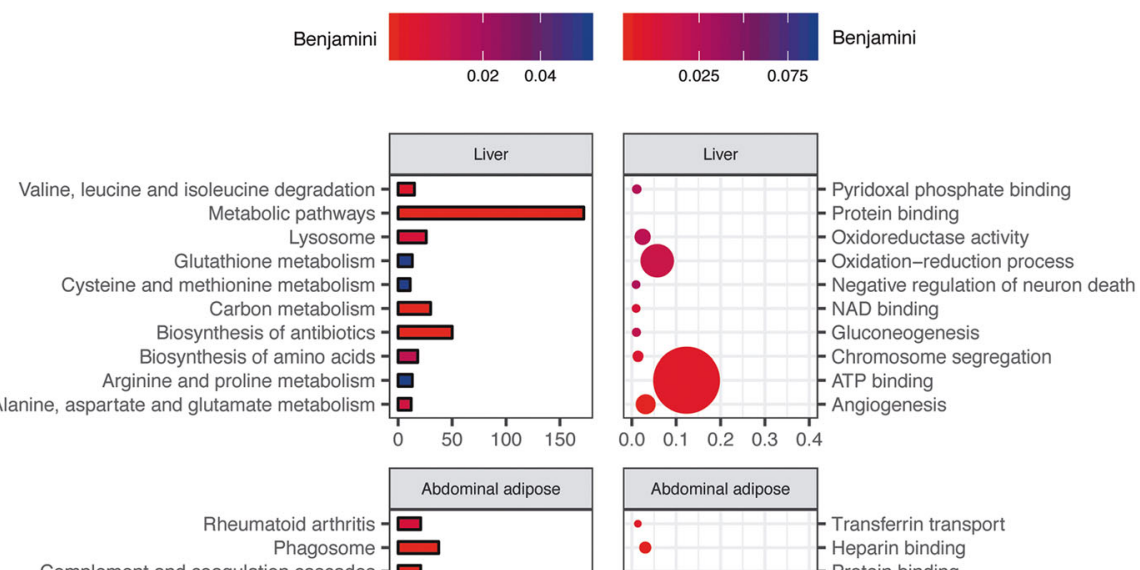

Complement and coagulation cascades

Phenylalanine, tyrosine and tryptophan biosynthesis -

$$
\text { Metabolic pathways - }
$$
Lysosome
Cysteine and methionine metabolism
Carbon metabolism Biosynthesis of antibiotics Biosynthesis of amino acids -
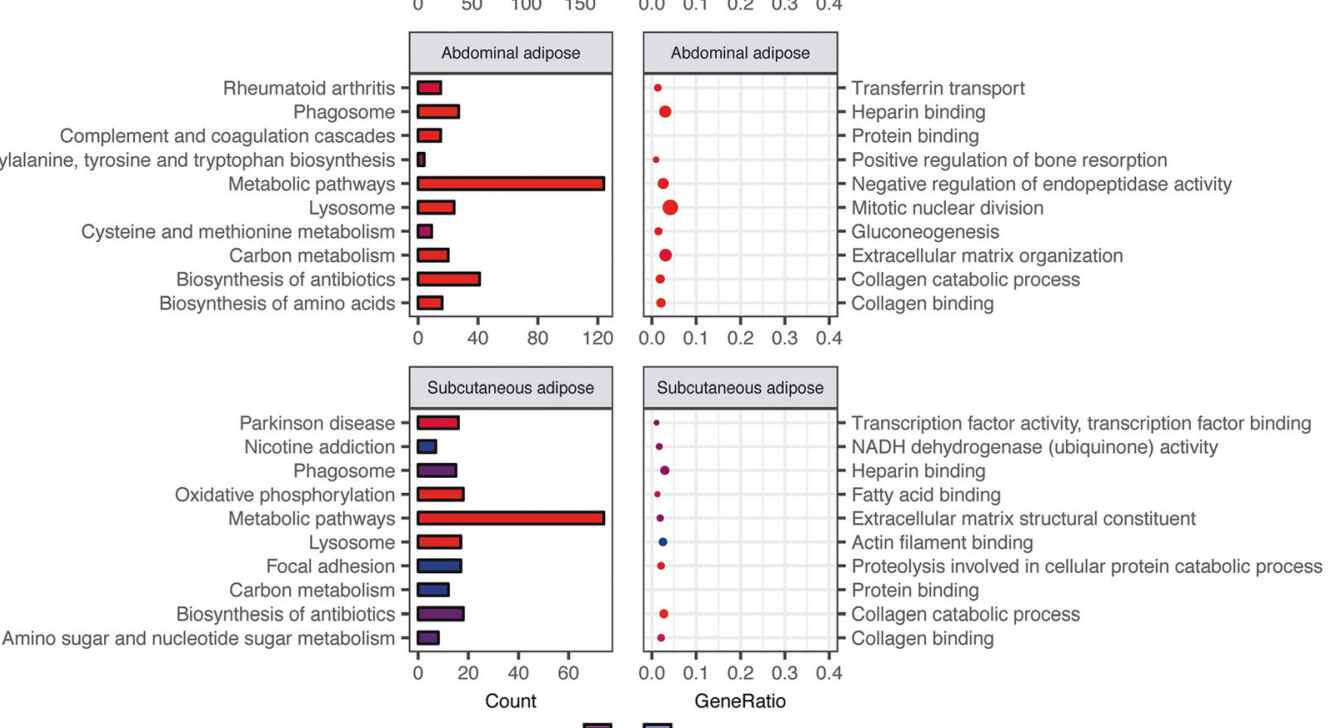

B
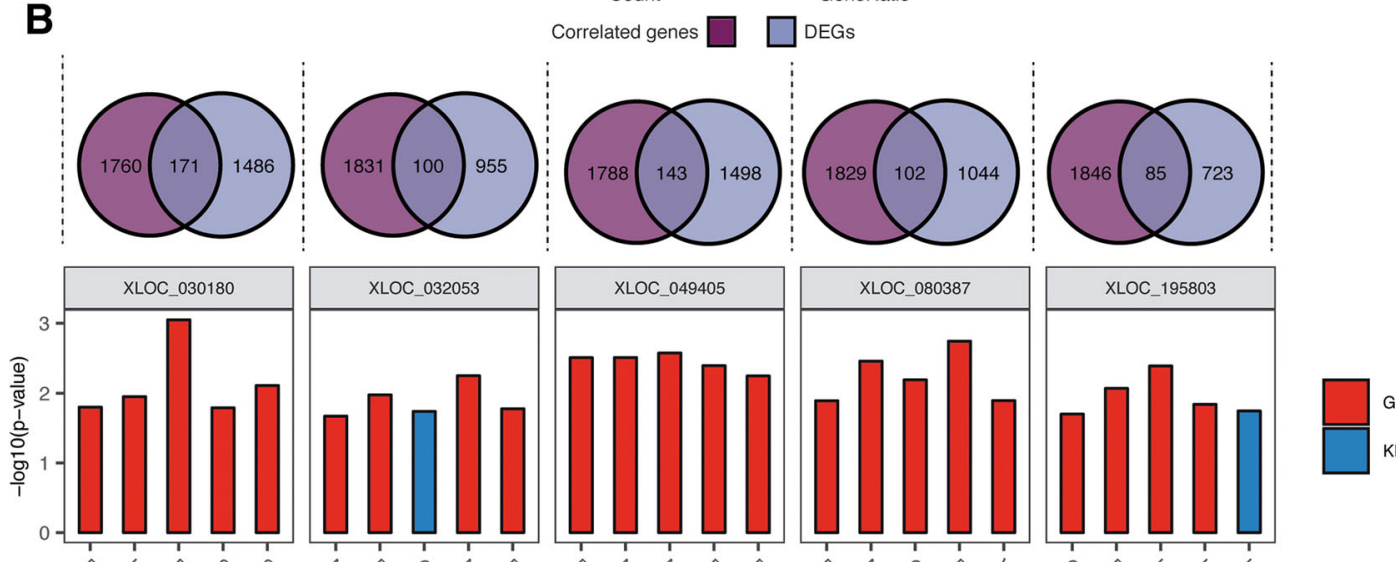

GO term

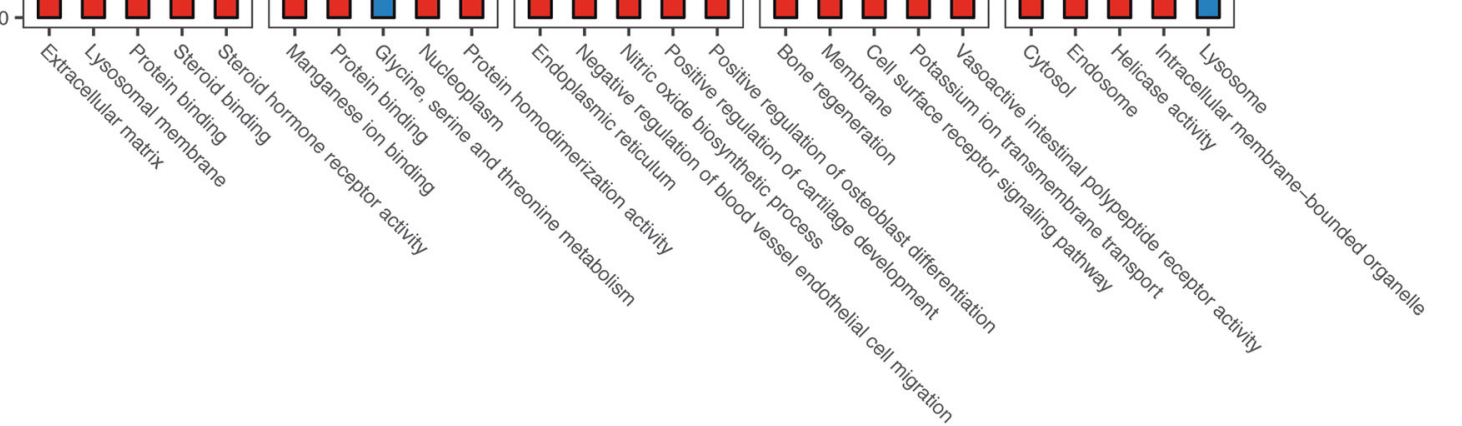

Fig. 3 (See legend on next page.) 
(See figure on previous page.)

Fig. 3 Enrichment of differentially expressed genes and IncRNA-correlated genes. a Top 10 GO/KEGG pathways of differentially expressed genes in liver, abdominal adipose and subcutaneous adipose. Left - enrichment of KEGG pathways, right - enrichment of GO terms. $\mathbf{b}$ Exhibition of the enrichment results of 5 IncRNAs correlated with differentially expressed genes (DEGs). Correlated genes were defined as genes associated with IncRNAs that have correlation coefficients over 0.80 and $p$-value $<0.05$

lncRNAs could provide more details about the regulatory functions of them.

\section{Discussion}

The accumulation of lipid, mostly triacylglycerol (TAG), within hepatocytes is the key prerequisite for the development of non-alcoholic fatty liver disease (NAFLD) in humans. Serum non-esterified fatty acids (NEFA) are the most dominant source for lipid accumulation in liver [3]. The major sources contributing to the level of serum NEFA include NEFA derived from fatty acid flux in adipose tissues and from chylomicrons through lipoprotein spillover [18]. Fatty acid flux in adipose tissues uses the de novo lipogenesis pathway to synthesize fatty acids from 2-carbon precursors made from dietary glucose. Research has shown non-alcoholic fatty liver disease patients have elevated masses of visceral and subcutaneous adipose tissues. Current therapeutic strategies for NAFLD patients are focusing on the reduction of adipose tissue fatty acid flux [19], indicating the important role of adipose tissues in the regulation of lipid metabolism.

Among goose adipose tissues and liver, our results showed that goose liver was the only lipogenic tissue with significantly increased weight (Fig. 1b). However, another study using Tianfu geese showed a significant increase in abdominal adipose weight [20]. Other research has shown breed differences in capability to deposit fat [21]. In our study, we only found an increasing trend for abdominal adipose. In earlier work, aside from weight change, the percentage of lipid content in adipose tissue did not significantly change, while the percentage of lipid content in liver increased significantly [20]. This is consistent with our results and in contrast to fat content accumulation in mammalian adipose, which is the prioritized tissue for lipid deposition. In fact, the obesity in human was widely considered as a metabolic syndrome in adipose tissues [22], suggesting the important role of adipose tissues in mammals. These results indicated that lipid deposition in goose under high-intake feeding is different from mammals and this distinct difference in lipid deposition in liver and adipose tissues under high-intake feeding, compared to normal intake, implies there may be an intrinsic difference in transcriptional regulation. This adaptation may be related to long-distance migration, because poultry species like duck have a similar ability, whereas terrestrial poultry like chicken do not deposit lipid in similar way [7]. Interestingly, leg muscle also showed a significant increase in weight. We hypothesize this is an adaptation to the increased weight during the feeding process and an increase in the amount of intramuscular fat [23, 24]. Further experiments need to be done to determine whether it is an increase in muscle cell volume or an increase in the amount of intramuscular fat.

By investigating the expression patterns of protein-coding genes, the phenotypic difference was also reflected by the transcriptome-wide patterns. The clear segregation of liver expression profiles in protein coding genes and the lack of a dominant pattern of expression among adipose profiles showed that there was distinct difference between goose livers but not adipose tissues after normal feeding and high-intake feeding (Fig. 2a). The higher average correlation coefficients between the two adipose tissues also showed the high similarity between geese from the two feeding groups (Fig. 2b). Similar results have been observed in chickens that showed larger changes in liver than adipose tissues [25].

However, what we found by clustering the transcriptome-wide expression patterns does not necessarily reveal which genes are the drivers of the responses to feeding treatment. In order to accommodate more energy, it is reasonable to expect an increase in metabolism with high-intake feeding. Indeed, through our differential expression analysis, we revealed that genes related to metabolism were upregulated in liver and adipose tissues under this feeding treatment. For example, glycolysis, the first major metabolic pathway that breaks down glucose, was intensely upregulated after high-intake feeding. Phophoglucomutase- 1 that controls the irreversible step of glycolysis was up-regulated [26]. Another important aspect about the formation of goose fatty liver is the balance between the storage and secretion of newly synthesized endogenous lipids and exogenous lipids in the plasma [7]. Key genes such as FADS1 and $A P O B$ in this process were regulated, leading to lipid deposition in liver. We found the elevated level of stearoyl-CoA desaturase $(S C D)$, an essential enzyme that transforms saturated fatty acids into unsaturated fatty acids [10], that was expressed nearly 3 -fold higher in liver for geese fed a high-intake diet. This gene was also expressed 1.7-fold higher in abdominal adipose for the same diet, but not significantly differentially expressed in subcutaneous adipose. In the goose reference genome sequence [7] there were more $S C D$ gene copies than chicken and expression of SCD was significantly increased after overfeeding. Moreover, we found FADS family and DGAT2, which 


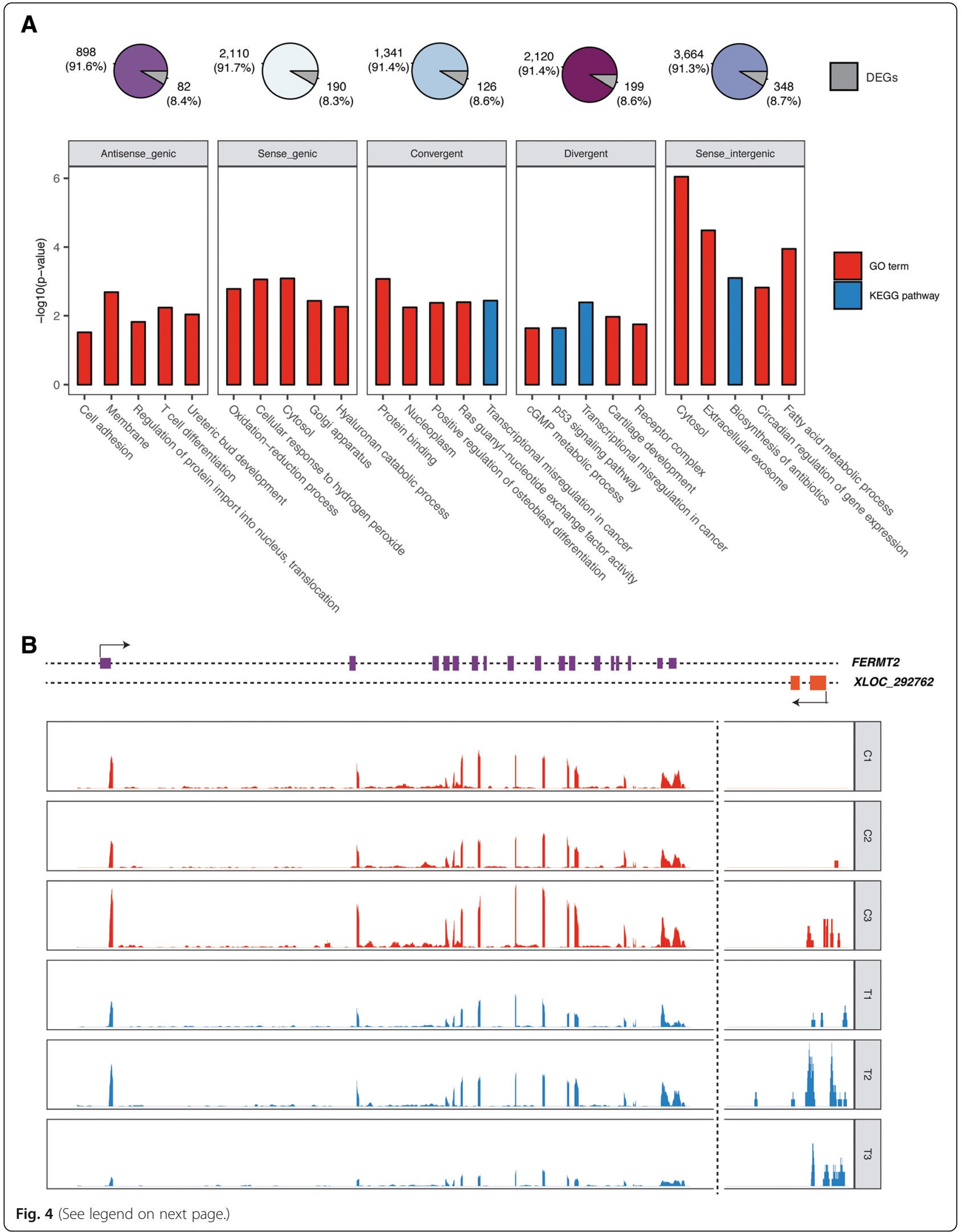


(See figure on previous page.)

Fig. 4 Prediction of IncRNA functions. a Enrichment of highly correlated mRNAs of different IncRNA types. LncRNAs were divided into 5 panels based on their type and were designated with a distinct color in the pie chart. Pie charts show the percentage of differentially expressed genes (DEGs) among correlated genes. $\mathbf{b}$ Exhibition of possible cis-acting IncRNA XLOC_292762 which locates at 5700 bp downstream. The expression scale of XLOC_292762 is different to FERMT2

both are involved in balancing lipid storage and degradation, were upregulated. This result demonstrates there is similarity between goose and mammals for lipid flux in adipose tissue, whereby the adipose tissue is contributing to the serum NEFA pool even though it may not be the major source of NEFA.

During the desaturation process mediated by $S C D$, two-carbon acetyl-CoA enters the citric acid cycle to generate ATP. The last few reactions of the citric acid cycle take place in mitochondria, instead of in the cytoplasm, and these steps are the final steps for beta-oxidation of fatty acids as well. Even though our results showed that genes from the mitochondrial genome were not differentially expressed, nuclear mitochondria-related genes were upregulated. These genes include $M D H 2$, which encodes mitochondria-located malate dehydrogenase 2 and plays a central role in the malate-aspartate shuttle [27]. Our results are consistent with previous research who has identified five nuclear mitochondria-related genes were up-regulated in goose fatty liver [28], and again, the differences in expression levels of these genes in liver, abdominal adipose tissue, and subcutaneous adipose tissue support our observation that liver played the key role in the regulation process.

Usually, uncontrolled NAFLD will turn into nonalcoholic steatohepatitis, which is a more severe disease with liver cell inflammation and cell damage [29]. Nonalcoholic steatohepatitis is also a precursor for liver cancer. In our results, we found an interesting down-regulation of some important cancer pathways such as the PI3K-Akt and cGMP-PKG signaling pathways. These pathways are largely involved in processes like tumorigenesis [30,31]. Our results showed the key genes of these pathways such as PI3K and PKG were down-regulated. Though it is too early to conclude the meaning of the down-regulation of these pathways, we believe our results provide evidence that goose develops little pathological development in fatty liver.

In addition to regulation by protein-coding genes, long noncoding RNAs have been recently identified in many species and function in many regulatory processes [32]. Typically, they are transcripts over $200 \mathrm{bp}$ and are typically expressed at lower levels than protein-genes in cells [33]. they have been demonstrated to be functional in many biological processes [13, 32-34] who tend to be involved in many processes as cis- or trans-acting regulators [13]. To thoroughly understand the transcriptomic changes, we identified lncRNAs in goose for the first time and showed their possible roles in lipid metabolism. Expression patterns of lncRNAs were similar to protein coding genes in liver and the expression patterns of IncRNAs remained unclustered in adipose tissues. Our functional prediction for IncRNAs revealed the high possibility that they are actively involved in lipid metabolism and other processes in response to high-intake feeding. LncRNAs show high variation between species, which makes them hard to annotate based on sequence. Current computational prediction of the function of lncRNAs is based on correlated expression and their genomic location relative to protein coding genes. Although further functional validation is needed, our research has provided some evidence for the role of lncRNAs in goose.

Recently, non-alcoholic fatty liver has been strongly associated with obesity in humans [35]. More than 1500 genes are strongly associated with obesity, in general, in mammals [36]. Among the more than 190 GO terms that have undergone rapid or slow evolution between goose and terrestrial birds based on previous research, GO terms including lipid binding, carbohydrate metabolic process and phosphatidylinositol phospholipase $\mathrm{C}$ activity were identified [7]. These results suggested that obesity-related genes may also be under selection to adapt to massive energy intake. There was a strong orthologous relationship between these obesity genes from mammals and goose genes (Fig. 5a), 1190 goose genes out of the 1518 mammalian genes identified to be orthologous to each other, the majority of orthologous genes shared a 1:1 orthologous relationship to human (74.66\%). We think this suggests that genes related to obesity showed more sequence-level variants rather than change in copy number. Additionally, obesity-related genes had a significantly higher proportion of DEGs (Chi-square $p$-value $\left.=4.42 * 10^{-4}\right)$, suggesting a possible functional regulation of obesity as well [36]. Most DEGs shared a 1:1 orthologous relationship with human (or chicken) and accounted for 18\% of all goose-human 1:1 obesity genes (or 18.49\% of goose-chicken 1:1 obesity genes) (Fig. 5b).

Given that we found $\sim 80 \%$ of obesity genes were $1: 1$ orthologs in chickens and humans, and the fact that domesticated goose does not develop pathological hepatic steatosis upon high-intake feeding, we believe fully understanding the genetic mechanism behind the formation of goose fatty liver will help to uncouple the effects of obesity and human non-alcoholic fatty liver. 


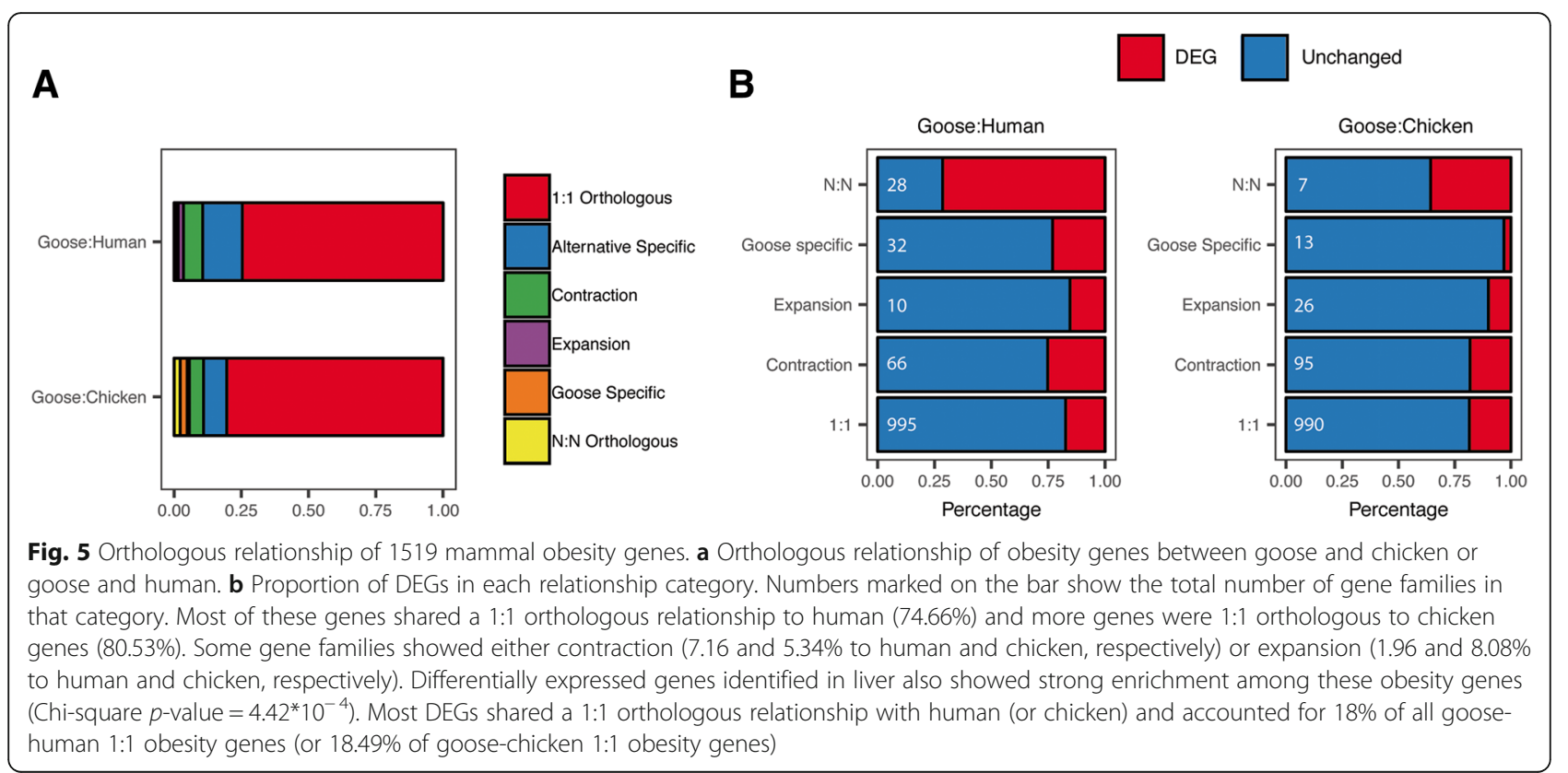

\section{Conclusions}

In this study, we found that the goose uses liver as the major tissue to regulate a distinct lipid synthesis and degradation flux. To process extra energy, the regulation of gene expression is stronger in goose liver than in adipose tissues. This is different from the expression profiles in mammals. We demonstrated by dynamic expression network analyses that there are numerous layers of positive responses to both massive energy intake and possible pathological development. The ability to deposit large amounts of fatty acids in liver instead of adipose tissues, and the upregulation of many metabolic genes need further analysis to reveal the genetic mechanism behind. Our results offer insights into goose adipogenesis and provide a new perspective for research in human metabolic dysregulation.

\section{Methods}

\section{Animals and their treatment}

All animal handling procedures were approved by $\mathrm{Si}$ chuan Agricultural University Animal Welfare Committee. Twenty healthy 126-day-old males of the Tianfu meat geese breed were obtained from Sichuan Agricultural University Waterfowls Breeding Farm. Tianfu is a lean Chinese commercial breed that is an $A$. anser x $A$. cygnoides hybrid. The breed is a composite of $87.5 \%$ Landes (A. anser) and $12.5 \%$ of Sichuan White (A. cygnoides). The population used in this study is closed and has been under selection for over 10 generations. We randomly assigned geese to two groups $(N=10)$. All geese had free access to water at all times. All geese were given the same diet $(14.29 \mathrm{MJ} / \mathrm{Kg}$ metabolizable energy, $8.09 \%$ crude protein and $0.14 \%$ methionine) but the two groups differed in daily feeding times. The control group had access to diet freely the whole day and on average consumed $325 \mathrm{~g}$ (energy level $4.64 \mathrm{MJ} / \mathrm{Kg}$ ) of feed a day. The feeding procedure for the high-intake feeding group was similar to what is used in the industry to produce foie gras. The birds were artificially force fed two times a day for days $1-3,3$ times a day for days $4-6$, 4 times a day for days $7-17$, and 3 times on day 18 . The meals on day 1 were $130 \mathrm{~g}$ and were increased by $5 \mathrm{~g}$ every day. By the end of the feeding period the difference in energy intake between the two group was $72.81 \mathrm{MJ}$ for each goose. On day 18 , the day before the birds were sacrificed, all geese were deprived of feed overnight and they were sacrificed the following morning at 145 days of age through cervical dislocation. Body weight was recorded every 3 days for each goose.

\section{Sample collection and total RNA-seq}

We collected liver, abdominal adipose, subcutaneous adipose, heart, breast muscle, leg muscle, duodenum, gizzard, glandular stomach, spleen and bursa of fabricius from each goose. Tissue weights were collected and tissue samples were put into liquid nitrogen immediately after being extracted from body.

For total RNA sequencing, we randomly selected 3 individuals as biological replicates from both the normal and the high-intake feeding group. Total RNA from liver, abdominal adipose, and subcutaneous adipose for each individual (a total of 18 samples) were extracted with the using RNeasy Mini Kit (QIAGEN, Germany) following the manufacturer's instructions. RNA integrity was checked by Agilent Bioanalyzer 2100 (Agilent Technologies, CA, USA). Samples with average RIN value = 7.59 (from 7 to 8.6) were then sent to Novogene 
(Tianjin, China) to generate paired-end libraries. All libraries were sequenced by Illumina Hiseq X 10 following Illumina's protocols by Novogene with a read length of $150 \mathrm{bp}$. Standard quality control and filtering of low quality reads was performed by Novogene and clean reads were provided for further analysis.

\section{Transcriptome alignment and assembly}

Clean reads were mapped against goose reference genome that includes the mitochondrial genome (AnsCyg_PRJNA183603_v1.0) using Bowtie2 [37] and the spliced reads aligner, Tophat2 [38] with default arguments. Tophat2 utilizes splicing information from the reference annotation file (GTF file) to guide the mapping of RNA-seq reads. BAM files for each library were then assembled by Cufflinks (V2.1.1) [39] using the $-g$ argument, which invokes assembly of transcripts based on the reference annotations, de novo assembly of transcripts using a probabilistic model, and quantifies the expression of assembled transcripts at the same time. Relative expression was reported as fragments per kilobase of transcripts per million mapped reads (FPKM). Cufflinks generated assembled transcripts in GTF file format for all individuals and these were later merged by tissue using Cuffmerge. For all annotated genes, Pearson's correlation coefficients of expression were calculated across the 18 samples. Principle variance components analysis (PVCA) was carried out using the R package pvca (https://www.bioconductor.org/ packages/release/bioc/html/pvca.html).

\section{Identification of long noncoding RNAs}

We used a homology-based method and constructed a personalized pipeline to identify putative long noncoding RNAs (lncRNAs). First, transcripts in the 6 merged GTF files (3 tissues $\times 2$ treatments) were compared to the reference annotations using Cuffcompare and those transcripts that were in the reference GTF file or had a large overlap with a known transcript were removed. Removed transcripts were marked as "c" or "=" by the custom Python script. By default, transcripts shorter than $200 \mathrm{bp}$ were not be retained. Protein-coding score were then calculated for remaining transcripts using CPC [40]. The protein-coding score is derived from comparison of a support vector machine learning classifier against a selected protein database. We calculated coding potential against UniRef 90 and transcripts with scores larger than 1 were filtered because of their high coding potential. Surviving transcripts were then scanned for potential of coding small peptides or functional protein domains using Infernal cmscan [41], which makes prediction using a Hidden Markov Model against a selected protein database. Here we used also UniRef 90 and again we filtered transcripts with high coding potential. Survived transcripts were then compared by blastx [42] to the NCBI NR database and UniRef 90 . We filtered any transcripts that only had one exon and retained the rest of them as putative lncRNAs. As we did for protein coding genes, we used Cufflinks to quantify the expression of the lncRNAs, and we performed principle variance components analysis and hierarchical clustering analysis.

\section{Identification of differentially expressed genes and functional enrichment analysis}

Cuffdiff was used to identify differentially expressed genes (DEGs) between tissues within the same treatment and between treatments for each tissue. Cuffdiff performs a pair-wise test for each pair of genes and corrects for multi-test bias at the same time using an FDR correction. Enrichment analysis between treatments was performed using the DAVID online enrichment tool (https://david.ncifcrf.gov) [43]. We first extracted the HGNC gene symbol for DEGs. We then converted HGNC symbols to human ENSEMBL IDs and applied these in DAVID. We performed the enrichment analysis for 3 gene ontology (GO) terms (biology process, molecular function, cellular component) and KEGG pathways. We separated enriched GO terms and KEGG pathways, and based on an adjusted Benjamini $p$-value, we selected the top 10 enriched GO terms and KEGG pathways for each tissue. We also separated DEGs into up-regulated and down-regulated DEGs and repeated the enrichment analysis.

\section{Analysis of putative IncRNAs}

We calculated the average length and number of exons of lncRNAs. Briefly, we categorized putative lncRNAs into sense intergenic lncRNAs, sense genic lncRNAs, convergent lncRNAs, divergent lncRNAs, and antisense intergenic lncRNAs [44]. As a prediction of possible functions of lncRNAs, we annotated the lncRNAs based on their proximity to nearby protein-coding genes. Specifically, lncRNAs that were within $10 \mathrm{~kb}$ upstream or downstream of a protein-coding gene were marked as possible cis-acting lncRNAs. Correlation analysis for expression of all genes and IncRNAs was performed by using the $\mathrm{R}$ package Hmisc (https://cran.r-project.org/ web/packages/Hmisc/). LncRNA and mRNA pairs with correlation coefficients $>0.80$ and $p<0.05$ were identified as correlated pairs. Customized python scripts were used to extract pairs that were both correlated and close to each other.

\section{Identification of orthologous gene families}

We downloaded the coding sequences (CDS) and peptide sequence of goose, human, and chicken from NCBI. When there was more than one alternative transcript of a gene, we only kept the transcript with the longest CDS 
and we filtered any transcripts that coded for fewer than 50 amino acids. Protein sequences from the final set of transcripts were subjected to BLAST [42] to obtain the similarity between sequences (e-value was set to $1^{*} 10^{-5}$ ). Hcluster_sg (https://github.com/douglasgscofield/hcluster) was used to cluster the results and T-Coffee [45] to rank the clusters. Finally, a customized script was used to categorize gene families (such as single-copy gene families, multi-copy gene families, and species-specific gene families).

\section{Additional files}

Additional file 1: Figure S1. Oil red staining of adipose tissues and integrated optical density of three tissues. (PDF 2908 kb)

Additional file 2: Figure S2. Comparison of characteristics of protein coding genes and IncRNAs and classification of IncRNAs. (PDF $163 \mathrm{~kb}$ )

Additional file 3: Figure S3. Principle components variance analysis of mRNA and IncRNA profiles. (PDF $509 \mathrm{~kb}$ )

Additional file 4: Figure S4. Pathway enrichment of up-regulated DEGs and down-regulated DEGs. (PDF 359 kb)

Additional file 5: Figure S5. Expression of mitochondria genes and important nuclear mitochondria-related genes. Asterisk mark indicates significantly differentially expressed between normal and high-dietary fed geese. (PDF $133 \mathrm{~kb}$ )

Additional file 6: Figure S6. GO enrichment of up-regulated/down-regulated DEGs. (PDF $806 \mathrm{~kb}$ )

Additional file 7: Table S1. Paired t-test of body weights between normal and high dietary group during the fattening process. (PDF $63 \mathrm{~kb}$ )

Additional file 8: Table S2. Paired t-test of relative tissue weight between normal and high dietary group. Relative weight = absolute weight/ body weight. (PDF $70 \mathrm{~kb}$ )

Additional file 9: Table S3. Top 10 highly correlated mRNA-IncRNA pairs within $10 \mathrm{~Kb}$ of each other. (PDF $77 \mathrm{~kb}$ )

\section{Abbreviations}

Akt: The serine-threonine protein kinase; ATP: Adenosine triphosphate; B3GALT2: Beta-1,3-galactosyltransferase 2; CDS: The coding sequence; CGMP: cyclic guanosine monophosphate; CPC: Coding potential calculator; DE: Differentially expressed; DEGs: Differentially expressed genes; FDR: False discovery rate; FERMT2: Fermitin family member 2; FPKM: Fragments per kilobase of exon per million reads; GJD2: Gap junction protein delta 2; GO: Gene ontology; GTF: Gene transfer format; HOXA10: Homeobox A10; kb: Kilobase; KEGG: Kyoto Encyclopedia of Genes and Genomes; IncRNA: long noncoding RNA; MDH2: Malate dehydrogenase 2; NAFLD: Non-alcoholic fatty liver disease; NEFA: Non-esterified fatty acids; PI3K: Phosphoinositide-3-Kinase; PKG: Protein kinase cGMP-dependent 1; PVCA: Principle variance components analysis; RIN: RNA integrity; SCD: Stearoyl-CoA desaturase; TAG: Triacylglycerol; ZFAT: Zinc finger and AT-hook domain containing

\section{Acknowledgements}

We thank Junsong Yuan for the help with goose sampling and tissue collecting experiments, Kranti Konganti for the help of debugging IncRNA identification pipeline.

\section{Funding}

This work was supported by grants from China Agricultural Research System (CARS-42-4), Project of National Science and Technology Plan for the Rural Development in China (No. 2015BAD03B06) to Dr. Jiwen Wang, for animal raising, phenotype and RNA-seq data collections, and the National Natural Science Foundation of China (31522055 and 31772576) to Dr. Mingzhou Li for the support of RNA-seq data collection and computational resource.

\section{Availability of data and materials}

All RNA-seg data generated and analyzed in this study have been deposited in NCBI's Gene Expression Omnibus under GEO Series accession number GSE119421. Additional supporting data can be accessed at https://figshare. com/s/cd02967c1991449d3b35.

\section{Authors' contributions}

GW, $L$ and $M L$ designed the study. GW, HX and $L J$ conducted the experiments. GW, QT, YL and SH analyzed the data. GW wrote the manuscript with input from $\mathrm{YL}, \mathrm{SH}$ and $\mathrm{LJ}$. ML, JW and CG supervised the study. All authors read and approved the final manuscript.

\section{Ethics approval and consent to participate}

All animal handling procedures were approved by Sichuan Agricultural University Animal Welfare Committee under DKY20150913.

\section{Consent for publication}

Not applicable.

\section{Competing interests}

The authors declare that they have no competing interests.

\section{Publisher's Note}

Springer Nature remains neutral with regard to jurisdictional claims in published maps and institutional affiliations.

\section{Author details}

${ }^{1}$ Farm Animal Genetic Resources Exploration and Innovation Key Laboratory of Sichuan Province, Sichuan Agricultural University, Chengdu, Sichuan 611130, People's Republic of China. ${ }^{2}$ Department of Animal Science, Texas A\&M University, College Station, TX 77843, USA.

Received: 16 November 2018 Accepted: 3 May 2019

Published online: 14 May 2019

\section{References}

1. Kershaw EE, Flier JS. Adipose tissue as an endocrine organ. J Clin Endocrinol Metab. 2004;89(6):2548-56

2. Aarsland A, Chinkes D, Wolfe RR. Hepatic and whole-body fat synthesis in humans during carbohydrate overfeeding. Am J Clin Nutr. 1997;65(6): 1774-82.

3. Meex RCR, Watt MJ. Hepatokines: linking nonalcoholic fatty liver disease and insulin resistance. Nat Rev Endocrinol. 2017:13(9):509-20.

4. Mourot J, Guy G, Peiniau P, Hermier D. Effects of overfeeding on lipid synthesis, transport and storage in two breeds of geese differing in their capacity for fatty liver production. Anim Res. 2006;55(5):427-42.

5. Hermier D, Salichon MR, Guy G, Peresson R. Differential channelling of liver lipids in relation to susceptibility to hepatic steatosis in the goose. Poult Sci. 1999:78(10):1398-406.

6. Xu HY, Wang Y, Han CC, Jiang L, Zhuo WH, Ye JQ, Wang JW. Estimation of lipoprotein-lipase activity (LPL) and other biochemical changes in two breeds of overfeeding geese. Asian Australas J Anim Sci. 2010;23(9):1221-8.

7. Lu L, Chen Y, Wang Z, Li X, Chen W, Tao Z, Shen J, Tian Y, Wang D, Li G, et al. The goose genome sequence leads to insights into the evolution of waterfowl and susceptibility to fatty liver. Genome Biol. 2015;16:89.

8. Webster RG, Bean WJ, Gorman OT, Chambers TM, Kawaoka Y. Evolution and ecology of influenza a viruses. Microbiol Rev. 1992;56(1):152-79.

9. Su SY, Dodson MV, Li XB, Li QF, Wang HW, Xie Z. The effects of dietary betaine supplementation on fatty liver performance, serum parameters, histological changes, methylation status and the mRNA expression level of Spot14alpha in Landes goose fatty liver. Comp Biochem Physiol A Mol Integr Physiol. 2009;154(3):308-14.

10. Pan ZX, Han CC, Wang JW, Li L, Tang H, Lv J, Lu L, Xu F. Cloning and expression of stearoyl-CoA desaturase 1 (SCD-1) in the liver of the Sichuan white goose and landes goose responding to overfeeding. Mol Biol Rep. 2011;38(5):3417-25.

11. Geng T, Xia L, Li F, Xia J, Zhang Y, Wang Q, Yang B, Montgomery S, Cui H, Gong D. The role of endoplasmic reticulum stress and insulin resistance in the occurrence of goose fatty liver. Biochem Biophys Res Commun. 2015; 465(1):83-7. 
12. Tang J, Fang Q, Shao R, Shen J, He J, Niu D, Lu L. Digital gene-expression profiling analysis of the fatty liver of Landes geese fed different supplemental oils. Gene. 2018;673:32-45.

13. Knoll M, Lodish HF, Sun L. Long non-coding RNAs as regulators of the endocrine system. Nat Rev Endocrinol. 2015;11(3):151-60.

14. Zhu H, Li B, Li L, Zhou L. Complete mitochondrial genome of swan goose Anser cygnoides (Anseriformes: Anatidae). Mitochondrial DNA A DNA Mapp Seq Anal. 2016;27(5):3177-8.

15. Tsunoda T, Shirasawa S. Roles of ZFAT in haematopoiesis, angiogenesis and cancer development. Anticancer Res. 2013;33(7):2833-7.

16. Han Y, Lu S, Wen YG, Yu FD, Zhu XW, Qiu GQ, Tang HM, Peng ZH, Zhou CZ. Overexpression of HOXA10 promotes gastric cancer cells proliferation and HOXA10(+)/CD44(+) is potential prognostic biomarker for gastric cancer. Eur J Cell Biol. 2015;94(12):642-52.

17. Lall VK, Bruce G, Voytenko L, Drinkhill M, Wellershaus K, Willecke K, Deuchars J, Deuchars SA. Physiologic regulation of heart rate and blood pressure involves connexin 36-containing gap junctions. FASEB J. 2017;31(9):3966-77.

18. Donnelly KL, Smith Cl, Schwarzenberg SJ, Jessurun J, Boldt MD, Parks EJ. Sources of fatty acids stored in liver and secreted via lipoproteins in patients with nonalcoholic fatty liver disease. J Clin Invest. 2005;115(5):1343-51.

19. Promrat K, Lutchman G, Uwaifo Gl, Freedman RJ, Soza A, Heller T, Doo E, Ghany M, Premkumar A, Park Y, et al. A pilot study of pioglitazone treatment for nonalcoholic steatohepatitis. Hepatology. 2004;39(1):188-96.

20. Han CC, Wang JW, Xu HY, Li L, Ye JQ, Jiang L, Zhuo WH. Effect of overfeeding on plasma parameters and mRNA expression of genes associated with hepatic lipogenesis in geese. Asian Australas J Anim Sci. 2008:21(4):590-5.

21. Davail S, Guy G, Andre J, Hermier D, Hoo-Paris R. Metabolism in two breeds of geese with moderate or large overfeeding induced liver-steatosis. Comp Biochem Physiol A Mol Integr Physiol. 2000;126(1):91-9.

22. Gesta S, Tseng YH, Kahn CR. Developmental origin of fat: tracking obesity to its source. Cell. 2007;131(2):242-56.

23. Fan B, Du ZQ, Rothschild MF. The fat mass and obesity-associated (FTO) gene is associated with intramuscular fat content and growth rate in the pig. Anim Biotechnol. 2009;20(2):58-70.

24. Hamdy O, Porramatikul S, Al-Ozairi E. Metabolic obesity: the paradox between visceral and subcutaneous fat. Curr Diabetes Rev. 2006;2(4):367-73.

25. Desert C, Baeza E, Aite M, Boutin M, Le Cam A, Montfort J, Houee-Bigot M, Blum Y, Roux PF, Hennequet-Antier C, et al. Multi-tissue transcriptomic study reveals the main role of liver in the chicken adaptive response to a switch in dietary energy source through the transcriptional regulation of lipogenesis. BMC Genomics. 2018;19(1):187.

26. Gloria-Bottini F, Magrini A, Antonacci E, La Torre M, Di Renzo L, De Lorenzo A, Bergamaschi A, Bottini E. Phosphoglucomutase genetic polymorphism and body mass. Am J Med Sci. 2007:334(6):421-5.

27. Minarik P, Tomaskova N, Kollarova M, Antalik M. Malate dehydrogenases-structure and function. Gen Physiol Biophys. 2002;21(3):257-65.

28. Osman RH, Shao D, Liu L, Xia L, Sun X, Zheng Y, Wang L, Zhang R, Zhang Y, Zhang J, et al. Expression of mitochondria-related genes is elevated in overfeeding-induced goose fatty liver. Comp Biochem Physiol B Biochem Mol Biol. 2016:192:30-7.

29. Spengler EK, Loomba R. Recommendations for diagnosis, referral for liver biopsy, and treatment of nonalcoholic fatty liver disease and nonalcoholic steatohepatitis. Mayo Clin Proc. 2015;90(9):1233-46.

30. Cully M, You H, Levine AJ, Mak TW. Beyond PTEN mutations: the PI3K pathway as an integrator of multiple inputs during tumorigenesis. Nat Rev Cancer. 2006;6(3):184-92.

31. Wong KK, Engelman JA, Cantley LC. Targeting the PI3K signaling pathway in cancer. Curr Opin Genet Dev. 2010;20(1):87-90.

32. Dey BK, Mueller AC, Dutta A. Long non-coding RNAs as emerging regulators of differentiation, development, and disease. Transcription. 2014; 5(4):e944014.

33. Iyer MK, Niknafs YS, Malik R, Singhal U, Sahu A, Hosono Y, Barrette TR, Prensner JR, Evans JR, Zhao S, et al. The landscape of long noncoding RNAs in the human transcriptome. Nat Genet. 2015;47(3):199-208.

34. Meng XY, Luo Y, Anwar MN, Sun Y, Gao Y, Zhang H, Munir M, Qiu HJ. Long non-coding RNAs: emerging and versatile regulators in host-virus interactions. Front Immunol. 2017;8:1663.

35. Ludwig J, Viggiano TR, McGill DB, Oh BJ. Nonalcoholic steatohepatitis: Mayo Clinic experiences with a hitherto unnamed disease. Mayo Clin Proc. 1980; 55(7):434-8
36. Kunej T, Jevsinek Skok D, Zorc M, Ogrinc A, Michal JJ, Kovac M, Jiang Z. Obesity gene atlas in mammals. J Genomics. 2013;1:45-55.

37. Langmead B, Salzberg SL. Fast gapped-read alignment with bowtie 2. Nat Methods. 2012:9(4):357-9.

38. Kim D, Pertea G, Trapnell C, Pimentel H, Kelley R, Salzberg SL. TopHat2: accurate alignment of transcriptomes in the presence of insertions, deletions and gene fusions. Genome Biol. 2013;14(4):R36.

39. Trapnell C, Williams BA, Pertea G, Mortazavi A, Kwan G, van Baren MJ, Salzberg SL, Wold BJ, Pachter L. Transcript assembly and quantification by RNA-Seq reveals unannotated transcripts and isoform switching during cell differentiation. Nat Biotechnol. 2010;28(5):511-5.

40. Kong L, Zhang Y, Ye ZQ, Liu XQ, Zhao SQ, Wei L, Gao G. CPC: assess the protein-coding potential of transcripts using sequence features and support vector machine. Nucleic Acids Res. 2007;35(Web Server issue:W345-9.

41. Nawrocki EP, Eddy SR. Infernal 1.1: 100-fold faster RNA homology searches. Bioinformatics. 2013;29(22):2933-5.

42. Altschul SF, Gish W, Miller W, Myers EW, Lipman DJ. Basic local alignment search tool. J Mol Biol. 1990;215(3):403-10.

43. Jiao X, Sherman BT, Huang d W, Stephens R, Baseler MW, Lane HC, Lempick RA. DAVID-WS: a stateful web service to facilitate gene/protein list analysis. Bioinformatics. 2012;28(13):1805-6.

44. Wucher V, Legeai F, Hedan B, Rizk G, Lagoutte L, Leeb T, Jagannathan V, Cadieu E, David A, Lohi H, et al. FEELnc: a tool for long non-coding RNA annotation and its application to the dog transcriptome. Nucleic Acids Res. 2017;45(8):e57.

45. Notredame C, Higgins DG, Heringa J. T-coffee: a novel method for fast and accurate multiple sequence alignment. J Mol Biol. 2000;302(1):205-17.

\section{Ready to submit your research? Choose BMC and benefit from:}

- fast, convenient online submission

- thorough peer review by experienced researchers in your field

- rapid publication on acceptance

- support for research data, including large and complex data types

- gold Open Access which fosters wider collaboration and increased citations

- maximum visibility for your research: over $100 \mathrm{M}$ website views per year

At $\mathrm{BMC}$, research is always in progress.

Learn more biomedcentral.com/submissions 